\title{
Eggshell membrane:A possible new natural therapeutic for joint and connective tissue disorders. Results from two open-label human clinical studies
}

Kevin J Ruff'

Dale P DeVore ${ }^{2}$

Michael D Leu ${ }^{3}$

Mark A Robinson ${ }^{4}$

'ESM Technologies, LLC, Carthage, MO, USA; ${ }^{2}$ Membrell, LLC, Carthage,

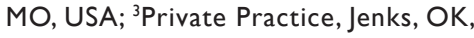
USA; ${ }^{4}$ Robinson Family Health Center, Carthage, MO, USA
Correspondence: Kevin J Ruff

ESM Technologies, LLC, 2213 Missouri St, Carthage, MO 64836, USA

Tel + I 4173584822

Fax + I 4173584954

Email kruff@esmingredients.com
This article was published in the following Dove Press journal:

Clinical Interventions in Aging

15 May 2009

Number of times this article has been viewed

Background: Natural Eggshell Membrane $\left(\mathrm{NEM}^{\circledR}\right)$ is a novel dietary supplement that contains naturally occurring glycosaminoglycans and proteins essential for maintaining healthy joint and connective tissues. Two single center, open-label human clinical studies were conducted to evaluate the efficacy and safety of $\mathrm{NEM}^{\circledR}$ as a treatment for pain and inflexibility associated with joint and connective tissue disorders.

Methods: Eleven (single-arm trial) and 28 (double-arm trial) patients received oral NEM ${ }^{\circledR}$ $500 \mathrm{mg}$ once daily for four weeks. The primary outcome measure was to evaluate the change in general pain associated with the treatment joints/areas (both studies). In the single-arm trial, range of motion (ROM) and related ROM-associated pain was also evaluated. The primary treatment response endpoints were at seven and 30 days. Both clinical assessments were performed on the intent-to-treat (ITT) population within each study.

Results: Single-arm trial: Supplementation with $\mathrm{NEM}^{\circledR}$ produced a significant treatment response at seven days for flexibility $(27.8 \%$ increase; $\mathrm{P}=0.038)$ and at 30 days for general pain (72.5\% reduction; $\mathrm{P}=0.007$ ), flexibility ( $43.7 \%$ increase; $\mathrm{P}=0.006$ ), and ROM-associated pain $(75.9 \%$ reduction; $\mathrm{P}=0.021)$. Double-arm trial: Supplementation with $\mathrm{NEM}^{\circledR}$ produced a significant treatment response for pain at seven days for both treatment arms (X: $18.4 \%$ reduction; $\mathrm{P}=0.021$. Y: $31.3 \%$ reduction; $\mathrm{P}=0.014$ ). There was no clinically meaningful difference between treatment arms at seven days, so the $\mathrm{Y}$ arm crossed over to the $\mathrm{X}$ formulation for the remainder of the study. The significant treatment response continued through 30 days for pain (30.2\% reduction; $\mathrm{P}=0.0001$ ). There were no adverse events reported during either study and the treatment was reported to be well tolerated by study participants.

Conclusions: Natural Eggshell Membrane $\left(\mathrm{NEM}^{\circledR}\right)$ is a possible new effective and safe therapeutic option for the treatment of pain and inflexibility associated with joint and connective tissue (JCT) disorders. Supplementation with $\mathrm{NEM}^{\circledR}, 500 \mathrm{mg}$ taken once daily, significantly reduced pain, both rapidly (seven days) and continuously (30 days). It also showed clinically meaningful results from a brief responder analysis, demonstrating that significant proportions of treated patients may be helped considerably from $\mathrm{NEM}^{\circledR}$ supplementation. The Clinical Trial Registration numbers for these trials are: NCT00750230 and NCT00750854.

Keywords: arthritis, pain, stiffness, eggshell membrane, joint, connective tissue, complimentary, alternative

\section{Background}

It is estimated that 140 million adults in the United States (US) suffer from some form of joint or connective tissue (JCT) disorder (ie, arthritis, lupus, gout, fibromyalgia, neck 
or back pain, etc. .) ${ }^{1,2}$ As the population ages, this estimate is expected to grow rapidly. Traditional treatments for most of these disorders attempt to address only the symptoms (pain, inflammation, and discomfort) associated with the diseases. This usually involves the use of analgesics (ie, acetaminophen, oxycodone, propoxyphene) or nonsteroidal anti-inflammatory drugs (NSAIDs) (ie, ibuprofen, diclofenac, celecoxib) alone or in combination. Most of these treatments have shown limited effectiveness in randomized controlled clinical trials (RCTs) ${ }^{3-6}$ or are known to have significant and sometimes severe side effects. To avoid the cardiac risks, ${ }^{7,8}$ gastrointestinal issues, ${ }^{9,10}$ and dependency issues ${ }^{11,12}$ associated with traditional JCT treatments (particularly with long-term use), many patients have turned to complementary and alternative medicines (CAMs) such as dietary supplements.

Glucosamine, chondroitin, and methylsulfonylmethane (MSM) alone and in combination, are widely marketed as dietary supplements to treat joint pain due to osteoarthritis (OA). There have been two major human clinical trials that have investigated the role of glucosamine and chondroitin in the treatment of OA symptoms. The Glucosamine/chondroitin Arthritis Intervention Trial (GAIT), a 1583 patient, six-month trial sponsored by the National Institutes of Health (NIH), failed to show significant improvement in the Western Ontario and McMasters Universities (WOMAC) Osteoarthritis Index in the overall patient population for glucosamine, chondroitin, or their combination. ${ }^{13}$ The Glucosamine Unum In Die (once-a-day) Efficacy (GUIDE) trial, a 318 patient, six-month European trial sponsored by industry, showed a small, $5 \%-6 \%$ improvement in total WOMAC Index score over placebo for glucosamine sulfate. ${ }^{14}$ Other vitamins, minerals, and botanicals such as kava, pine bark extract, capsicum, boswellia root extract, turmeric/curcumin, etc., are also marketed for various JCT pain maladies often with little or no clinical support. Because of their limited effectiveness, the search for additional CAMs to treat JCT disorders continues.

We present here the use of eggshell membrane as a possible new natural therapeutic for JCT disorders. Eggshell membrane is primarily composed of fibrous proteins such as collagen type I. ${ }^{15}$ However, eggshell membranes have also been shown to contain glycosaminoglycans, such as dermatan sulfate and chondroitin sulfate ${ }^{16}$ and sulfated glycoproteins including hexosamines, such as glucosamine. ${ }^{17}$ Other components identified in eggshell membranes are hyaluronic acid, ${ }^{18}$ sialic acid, ${ }^{19}$ desmosine and isodesmosine,${ }^{20}$ ovotransferrin, ${ }^{21}$ lysyl oxidase, ${ }^{22}$ lysozyme, ${ }^{23}$ and $\beta$-N-acetylglucosaminidase.$^{24}$ The discovery of eggshell membrane as a natural source of combined collagen, glucosamine, chondroitin, and hyaluronic acid has prompted the evaluation of this material as a potential treatment for joint and connective tissue pain. ESM Technologies, LLC (Carthage, MO, USA) has developed methods to efficiently and effectively separate eggshell membrane from eggshells to create an essentially shell-free eggshell membrane. The isolated membrane is then partially hydrolyzed using a proprietary process and dry-blended to produce $100 \%$ pure Natural Eggshell Membrane $\left(\mathrm{NEM}^{\circledR}\right)$ powder. Compositional analysis of $\mathrm{NEM}^{\circledR}$ conducted by ESM has identified a high content of protein and moderate quantities of glucosamine (up to $1 \%$ by dry weight), chondroitin sulfate (up to $2 \%$ ), hyaluronic acid (up to $2 \%$ ), and collagen (Type I, up to $25 \%$ ).

Two 1-month pilot clinical studies were conducted to evaluate the efficacy and safety of $\mathrm{NEM}^{\circledR}$ for the relief of the pain and discomfort associated with joint and connective tissue disorders. The results are presented herein.

\section{Methods}

\section{Study designs}

Both studies were conducted according to a single center, open-label, controlled design and were conducted in the US in accordance with the US Food and Drug Administration's principles of Good Clinical Practice (Title 21, Code of Federal Regulations, Parts 50 and 56 and ICH E6) and the Declaration of Helsinki. The study protocols were approved by an independent institutional review board (The Copernicus Group) and patients provided their written informed consent to participate. Subjects were required to suspend all current pain relief medications in order to participate in the study. For the double-arm trial, eligible subjects were then randomized either to $\mathrm{NEM}^{\circledR}$ formulation X or $\mathrm{NEM}^{\circledR}$ formulation $\mathrm{Y}$ treatment groups in the order in which they were enrolled in the study using a block randomization table. $\mathrm{NEM}^{\circledR}$ formulations $\mathrm{X}$ and $\mathrm{Y}$ differed in the degree $(\mathrm{Y} \sim 2.5 \times>\mathrm{X}$ ) to which they were partially hydrolyzed prior to final ingredient blending. The clinical investigators were not blinded to treatment (open label) (both studies) or randomization (double-arm trial), however the subjects were blinded to randomization in the double-arm trial. Treatment consisted once daily orally of either $\mathrm{NEM}^{\circledR}$ (Membrell, LLC, Carthage, MO, USA) (single-arm trial) or two different formulations of $\mathrm{NEM}^{\circledR}$ (double-arm trial) provided in $500 \mathrm{mg}$ vegetarian capsules that were stored in closed containers at ambient temperature. Clinic visits were scheduled for subjects at seven and 30 days following the onset of treatment. Treatment compliance was checked at clinic visits by patient interview and by counting the number of unused doses of the study medications. Although discouraged, acetaminophen was allowed for pain 
relief rescue, if necessary. Subjects recorded the time and amount of acetaminophen taken in patient diaries.

\section{Patients}

All subjects aged 18 years or older and seeking relief of mild to moderate pain due to joint or connective tissue disorders were considered for enrollment in the studies. In order to be eligible, subjects must have had persistent pain (lasting at least three months) associated with a JCT disorder, with a minimum baseline pain level of 2 on a 0 to 10 analog Likert scale. Subjects were required to suspend all current pain relief medications. Subjects that were currently taking analgesic medications were eligible to participate in the studies following a 14-day washout period for NSAIDs, a seven-day washout for narcotics, and a 90-day washout for injected steroids. Subjects currently taking glucosamine, chondroitin sulfate or MSM were only eligible after a three-month washout period. Patients were excluded if they were currently receiving remission-inducing drugs such as methotrexate or immunosuppressive medications or had received them within the past three months. Other exclusionary criteria were: body weight 113.5 kilograms (250 lbs.) or greater, a known allergy to eggs or egg products, or pregnant or breastfeeding women. Subjects previously enrolled in a study to evaluate pain relief within the past six months or currently involved in any other research study involving an investigational product (drug, device, or biologic) or a new application of an approved product, within 30 days of screening were also excluded from participating in the trials.

\section{Treatment response}

\section{Single-arm trial}

The primary outcome measure of this study was to evaluate the mean effectiveness of $\mathrm{NEM}^{\circledR}$ in relieving general pain associated with moderate JCT disorders. Additional primary outcome measures were to evaluate flexibility, as well as the pain associated with the range of motion (ROM) evaluation. The primary treatment response endpoints were the 7 and 30 day clinic assessments utilizing a 0 to 10 analog Likertscale, with 0 equating to no pain and 10 equating to most severe pain. Patients were asked to record a number equating to the perceived pain from the treatment joint/area. Endpoints were then compared to pretreatment assessments.

\section{Double-arm trial}

The primary outcome measure of this study was to evaluate the mean effectiveness of $\mathrm{NEM}^{\circledR}$ in relieving general pain associated with moderate JCT disorders. Subjects were allowed to evaluate multiple treatment joints/areas. The primary treatment response endpoints were the seven and 30 day clinic assessments utilizing a 0 to 10 analog Likert scale, with 0 equating to no pain and 10 equating to most severe pain. Patients were asked to record a number equating to the perceived pain from the treatment joints/areas. Endpoints were then compared to pretreatment assessments.

\section{Adverse events}

Secondary objectives of both studies were to evaluate tolerability and any adverse reactions associated with supplementation with $\mathrm{NEM}^{\circledR}$. The subjects' self-assessment diaries were reviewed and any discomfort or other adverse events were recorded and reported in accordance with applicable FDA regulations. Adverse events and serious adverse events were assessed by the clinical investigator at each study visit and followed until resolution, as necessary. Serious adverse events (those that result in either death, a life-threatening experience, inpatient hospitalization, a persistent or significant disability or incapacity; or require, based on reasonable medical judgment, a medical or surgical intervention to prevent an outcome described previously) were required to be reported to the clinical monitor immediately.

\section{Statistical analysis}

As these were pilot studies with nonspecified treatment joints/areas, no enrollment sample size estimates were performed for statistical power determination. Comparisons of the two treatment groups (double-arm trial) were made with a two-sided independent group $t$-test at baseline to validate randomization. Statistical significance was accepted at an $\alpha$ value of $<0.05$. Post-baseline statistical analyses were done as pairwise comparisons utilizing a two-sided $t$-test. Statistical significance was accepted at an $\alpha$ value of $<0.05$. Analysis of the primary outcome measure (the change from baseline in general pain levels (both studies), as well as flexibility and ROM-associated pain (single-arm trial)) was conducted in the intent-to-treat (ITT) population (ie, including all patients with at least one efficacy assessment). The last observation carried forward (LOCF) approach was used for patients who made at least one follow-up visit but who did not complete the study (lost to follow-up). A per-protocol completer analysis was also performed. SYSTAT software (version 12) was used for all statistical analyses. ${ }^{25}$

\section{Results}

\section{Single-arm trial}

Patient recruitment began in October 2003 at a single clinical site in Missouri (USA) and the final follow-up was conducted in December 2003. A total of 11 subjects were enrolled with 
various joint and connective tissue conditions. Of these subjects, five $(45 \%)$ were female and six $(55 \%)$ were male. The treatment joints/areas consisted of knees (3), hips (1), elbows (1), neck (1), shoulders (1), and lower back (4). All 11 subjects completed baseline evaluations and were therefore used as the ITT population. Ten (91\%) of the 11 ITT subjects completed the one-month study per the protocol. Of the 11 subjects in the ITT population, one subject did not begin treatment and, therefore, was not available for further analysis. Compliance with the study treatment regimen was good in the treatment group. In those subjects that completed the study, the rate of compliance was $>98 \%$ (as judged by capsule count at clinic visits).

A clinical comparison of valid subjects was carried out to obtain a mean baseline pain value for the study population of $3.6 \pm 1.8$ (mean \pm standard deviation), a mean flexion ROM of $64.2^{\circ} \pm 36.5^{\circ}$, and a mean ROM-associated pain value of $2.9 \pm 2.6$. Statistical analysis of the primary outcome measures revealed that supplementation with $\mathrm{NEM}^{\circledR}$ produced a significant treatment response at seven days for flexibility (27.8\% increase; $\mathrm{P}=0.038$ ) and at 30 days for general pain (72.5\% reduction; $\mathrm{P}=0.007)$, flexibility $(43.7 \%$ increase; $\mathrm{P}=0.006)$, and ROM-associated pain $(75.9 \%$ reduction; $\mathrm{P}=0.021$ ) (see Table 1). Overall, the use of rescue pain medication was extremely low throughout the study, occurring once in every 20-22 days.

\section{Double-arm trial}

Patient recruitment began in November 2003 at a single clinical site in Missouri (USA) and the final follow-up was conducted in February 2004. A total of 28 subjects were enrolled with various joint and connective tissue conditions, some with multiple treatment joints/areas. Of these subjects, six (21\%) were male and $22(79 \%)$ were female. The treatment joints/areas consisted of knees (7), hips (8), neck (1), shoulders (9), hands (2), legs (1), feet (1), lower back (4), and nonspecific (3). All 28 subjects completed baseline evaluations and were therefore used as the ITT population. Fourteen subjects $(50 \%)$ were randomized to the $\mathrm{NEM}^{\circledR} \mathrm{X}$ formulation treatment group and 14 subjects $(50 \%)$ were randomized to the $\mathrm{NEM}^{\circledR} \mathrm{Y}$ formulation treatment group. Twenty (71\%) of the 28 ITT subjects completed the one-month study per the protocol. Of the 28 subjects in the ITT population, two subjects assigned to the $\mathrm{X}$ arm did not begin treatment and, therefore, were not available for further analysis. One patient in the $\mathrm{X}$ arm and 1 patient in the $\mathrm{Y}$ arm officially withdrew from the study due to lack of efficacy. The overall drop-out rate of $29 \%$ (from enrollment) was higher than expected, although $77 \%$ of the subjects that began treatment $(n=26)$ completed the study. Compliance with the study treatment regimen was good in both treatment groups. In those subjects that completed the study, the rate of compliance was $>96 \%$ (as judged by capsule count at clinic visits).

Patient data was initially evaluated to ensure randomization between groups $(\mathrm{P}=0.097)$. A clinical comparison of valid subjects was carried out to obtain a mean baseline pain value (mean \pm standard deviation) for each $\operatorname{arm}(\mathrm{X}$ and $\mathrm{Y}$ ) of the study (X: $6.8 \pm 1.9 ; \mathrm{Y}: 5.6 \pm 1.9$ ). Statistical analysis of the primary outcome measures revealed that supplementation with $\mathrm{NEM}^{\circledR}$ produced a significant rapid treatment response for pain at seven days for both treatment arms $(\mathrm{X}: 18.4 \%$ reduction; $\mathrm{P}=0.021$. $\mathrm{Y}: 31.3 \%$ reduction; $\mathrm{P}=0.014)$. The $12.9 \%$ difference between treatment arms was not as large as was expected from the difference in the formulations. It was decided that this difference was not clinically meaningful. Therefore, at seven days the $\mathrm{Y}$ arm crossed over to the $\mathrm{X}$ formulation for the remainder of the study. The significant treatment response continued through 30 days for pain $(30.2 \%$ reduction; $\mathrm{P}=0.0001)$ (See Table 2$)$. Overall, the use of rescue pain medication was extremely low throughout the study, occurring once in every 14-16 days. There were no significant differences between the treatment arms for rescue medication use.

Both study populations were too small to stratify the patients according to covariates, such as gender or treatment joint/area, to obtain statistically relevant data. There were no adverse events or serious adverse events reported during either of the studies. Of particular note is that there were no allergyassociated adverse events during the studies, although those with known egg allergies were excluded from participating during screening. In general, the treatment was reported to be extremely well tolerated by study participants.

\section{Discussion}

Joint and connective tissue disorders are extremely common in the United States and result in significant costs, both financial and quality of life, for those that suffer from the debilitating diseases. These two pilot clinical trials were designed to evaluate the efficacy and safety of $\mathrm{NEM}^{\circledR}$ as a treatment option for JCT disorders. Results from both pilot studies suggest that $\mathrm{NEM}^{\circledR}$ is both effective and safe for treating pain associated with JCT disorders and considerably improves flexibility in the affected joints/areas. $\mathrm{NEM}^{\circledR}$ has the added benefit of avoiding the concerning side effects associated with long-term use of other JCT treatments, such as narcotics or NSAIDs.

Patients experienced relatively rapid (seven days) responses for pain (double-arm trial) with a mean response of approximately 25\% (X: 18.4\%; Y: 31.3\%) and flexibility (single-arm trial) with a mean response of approximately $28 \%$. 
Table I Single-arm trial mean values by category at baseline, 7 and 30 days post-treatment

\begin{tabular}{lllll}
\hline & Days post-treatment & Mean \pm SD & Percent improvement & P-value \\
\hline General Pain & Baseline $(\mathrm{n}=\mathrm{II})$ & $3.6 \pm 1.8$ & - & - \\
& $7(\mathrm{n}=\mathrm{II})$ & $2.7 \pm 1.7$ & $25.8 \%$ & $0.5 \mathrm{I} 5$ \\
& $30(\mathrm{n}=\mathrm{II})$ & $1.0 \pm 1.2$ & $72.5 \%$ & $* 0.007$ \\
Flexion (ROM) & Baseline $(\mathrm{n}=\mathrm{II})$ & $64.2^{\circ} \pm 36.5^{\circ}$ & - & - \\
& $7(\mathrm{n}=\mathrm{II})$ & $82.0^{\circ} \pm 41.4^{\circ}$ & $27.8 \%$ & $* 0.038$ \\
& $30(\mathrm{n}=\mathrm{II})$ & $92.2^{\circ} \pm 38.4^{\circ}$ & $43.7 \%$ & - \\
ROM Pain & Baseline $(\mathrm{n}=\mathrm{II})$ & $2.9 \pm 2.6$ & - & $0.1 \%$ \\
& $7(\mathrm{n}=\mathrm{II})$ & $1.7 \pm 2.1$ & $43.3 \%$ & $* 0.02 \mathrm{I}$ \\
\hline
\end{tabular}

Notes: $P$-values were determined by pairwise, two-sided, $t$-test comparison, and represent treatment versus baseline. ${ }^{*} P<0.05$.

Abbreviations: ROM, range of motion; SD, standard deviation.

The single-arm trial failed to reach statistical significance at seven days for pain. This is likely a result of the relatively high standard deviation at baseline (1.8) compared to mean pain at baseline (3.6). Each arm of the double-arm trial had a similar number of patients (X: 12; Y: 14) as in the single-arm trial (10) and yet had statistically significant results for pain at seven days. However the baseline means (X: 6.8; Y: 5.6) and standard deviations (both 1.9) were relatively better proportioned than the single-arm trial. The use of the pairwise t-test helps to mitigate the effects of a high standard deviation in determining statistical significance.

By the end of the follow-up period (30 days) the mean response for pain had improved to 30\% (double-arm trial) and $73 \%$ (single-arm trial). At the same time, flexibility improved to a mean response of approximately $44 \%$ and the ROM-associated pain had a mean response of approximately $76 \%$ (singlearm trial). A brief responder analysis of the data provides a number of clinically relevant highlights. In both the single-arm trial and the double-arm trial, a significant proportion of the study populations ( $64 \%$ and $35 \%$, respectively) experienced a greater than $50 \%$ reduction in pain by 30 days. Of particular note is that nearly half (45\%) of the patients in the single-arm trial reported that they were pain-free (reported a score of 0 ) by 30 days of supplementation. All patients in the single-arm trial experienced at least some improvement in flexibility or
ROM-associated pain, with more than half (55\%) of the subjects experiencing a greater than $50 \%$ improvement in flexibility and more than one-third $(36 \%)$ of the subjects reporting that they were pain-free during ROM evaluation. The safety profile for $\mathrm{NEM}^{\circledR}$ is also of significance as there are no known side effects, excluding the obvious egg allergy concern. This is of obvious importance in a condition that requires long-term treatment such as JCT disorders. Significant and sometimes serious side effects associated with other treatments can force patients to have to make the difficult decision between living with the disease symptoms or living with the side effect symptoms.

Both trials suffered from a limited initial enrollment (11 and 28 subjects), however both trials had relatively low drop-out rates $(9 \%$ and $29 \%)$. As both trials were also open label, there is the obvious issue of the placebo effect. The inclusion of a placebo control would have provided greater clinical meaning, however it would have required a significantly larger study population. The inclusion of such a broad range of JCT disorders with vastly differing pathologies could have readily led to ambiguous treatment effects. Additionally, a broad spectrum of disease severity was included in both trials which could have also led to ambiguous results. Despite these potential pitfalls, the results from these open-label trials suggest that $\mathrm{NEM}^{\circledR}$ may be an effective therapeutic for a broad range of JCT disorders. A larger study with some modifications is clearly warranted.

Table 2 Double-arm trial mean pain values at baseline, 7 and 30 days post-treatment

\begin{tabular}{lllll}
\hline Days post-treatment & X Mean \pm SD & Y Mean \pm SD & Percent improvement & P-value \\
\hline Baseline $(n=12,14)$ & $6.8 \pm 1.9$ & $5.6 \pm 1.9$ & - & - \\
$7(n=12,14)$ & $5.5 \pm 2.0$ & $3.9 \pm 2.5$ & $18.4 \%, 31.3 \%$ & $* 0.021, * 0.014$ \\
$30(n=26)$ & $4.3 \pm 2.3$ & - & $30.2 \%$ & $* 0.0001$ \\
\hline
\end{tabular}

Notes: $P$-values were determined by pairwise, two-sided, $t$-test comparison, and represent treatment versus baseline. $* P<0.05$.

Abbreviation: SD, standard deviation. 


\section{Conclusion}

With so many people suffering from joint and connective tissue disorders, and that number expected to grow immensely as the overall US population ages, it is important for patients to have treatment options that are both effective and safe. The reporting of the results from these two open-label pilot studies demonstrates that $\mathrm{NEM}^{\circledR}$ may be a viable treatment option for the management of JCT disorders. In these clinical studies, $\mathrm{NEM}^{\circledR}, 500 \mathrm{mg}$ taken once daily, significantly reduced pain, both rapidly (seven days) and continuously (30 days). It also showed clinically meaningful results from a brief responder analysis, demonstrating that a significant proportion of treated patients may benefit from $\mathrm{NEM}^{\circledR}$ supplementation.

\section{Disclosure}

KJR is currently employed by the sponsor of both studies. DPD has served as a paid consultant for the sponsor of the studies. MDL and MAR have no competing interests. KJR carried out the statistical analysis of the data and drafted the manuscript. DPD participated in the design of both studies, assisted in the statistical analysis of the data, and assisted in drafting the manuscript. MDL was the clinical investigator for the Double-Arm Trial and assisted in drafting the manuscript. MAR was the clinical investigator for the Single-Arm Trial and assisted in drafting the manuscript. All authors read and approved the final manuscript. The authors would like to acknowledge Dr Barry W. Ritz, Drexel University, Department of Bioscience and Biotechnology, USA, for his assistance with the preparation of this manuscript. Both studies were sponsored by ESM Technologies, LLC.

\section{References}

1. Helmick CG, Felson DT, Lawrence RC, et al. Estimates of the Prevalence of Arthritis and Other Rheumatic Conditions in the United States. Part I. Arthritis Rheum. 2008;58(1):15-25.

2. Lawrence RC, Felson DT, Helmick CG, et al. Estimates of the prevalence of arthritis and other rheumatic conditions in the United States. Part II. Arthritis Rheum. 2008;58(1):26-35.

3. Altman RD. Ibuprofen, acetaminophen and placebo in osteoarthritis of the knee: a six-day double-blind study [abstract]. Arthritis Rheum. 1999; 42:S403.

4. Case JP, Baliunas AJ, Block JA. Lack of efficacy of acetaminophen in treating symptomatic knee osteoarthritis: A randomized, double-blind, placebo-controlled comparison trial with diclofenac sodium. Arch Intern Med. 2003;163:169-178.

Clinical Interventions in Aging

\section{Publish your work in this journal}

Clinical Interventions in Aging is an international, peer-reviewed journal focusing on evidence-based reports on the value or lack thereof of treatments intended to prevent or delay the onset of maladaptive correlates of aging in human beings. This journal is indexed on PubMed Central, MedLine, the American Chemical Society's 'Chemical
5. Geba GP, Weaver AL, Polis AB, Dixon ME, Schnitzer TJ. Efficacy of rofecoxib, celecoxib, and acetaminophen in osteoarthritis of the knee: a randomized trial. JAMA. 2002;287(1):64-71.

6. Towheed TE, Maxwell L, Judd MG, Catton M, Hochberg MC, Wells G. Acetaminophen for osteoarthritis. Cochrane Database Syst Rev. 2006;1:CD004257.

7. Singh G, Wu O, Langhorne P, Madhok R. Risk of acute myocardial infarction with nonselective non-steroidal anti-inflammatory drugs: a meta-analysis. Arthritis Res Ther. 2006;8(5):153-162.

8. Solomon SD, McMurray JJ, Pfeffer MA, et al. Cardiovascular risk associated with celecoxib in a clinical trial for colorectal adenoma prevention. N Engl J Med. 2005;352:1071-1080.

9. Deeks JD, Smith LA, Bradley MD. Efficacy, tolerability, and upper gastrointestinal safety of celecoxib for treatment of osteoarthritis and rheumatoid arthritis: systematic review of randomised controlled trials. Brit Med J. 2002;325:619-627.

10. Laine L. Nonsteroidal anti-inflammatory drug gastropathy. Gastrointest Endosc Clin N Am. 1996;6(3):489-504

11. Porter J, Jick H. Addiction rate in patients treated with narcotics. N Engl J Med. 1980;320:123-126.

12. Weaver M, Schnoll S. Addiction issues in prescribing opioids for chronic nonmalignant pain. J Addict Med. 2007;1(1):2-10.

13. Clegg DO, Reda DJ, Harris CL, et al. Glucosamine, chondroitin sulfate, and the two in combination for painful knee osteoarthritis. $N$ Engl J Med. 2006;354(8):795-808.

14. Herrero-Beaumont G, Ivorra JAR, Trabado MC, et al. Glucosamine sulfate in the treatment of knee osteoarthritis symptoms. Arthritis Rheum. 2007;56(2):555-567.

15. Wong M, Hendrix MJC, von der Mark K, Little C, Stern R. Collagen in the egg shell membranes of the hen. Dev Biol. 1984;104(1):28-36.

16. Baker JR, Balch DA. A study of the organic material of hen's-egg shell. Biochem J. 1962;82:352-361.

17. Picard J, Paul-Gardais A, Vedel M. Sulfated glycoproteins from egg shell membranes and hen oviduct. Isolation and characterization of sulfated glycopeptides. Biochim Biophys Acta. 1973;320:427-441.

18. Long FD, Adams RG, DeVore DP. Preparation of hyaluronic acid from eggshell membrane. US Patent \#6946551, September 20, 2005.

19. Nakano T, Ikawa NI, Ozimek L. Chemical composition of chicken eggshell and shell membranes. Poult Sci. 2003;82:510-514.

20. Starcher BC, King GS. The presence of desmosine and isodesmosine in eggshell membrane protein. Connect Tissue Res. 1980;8(1): 53-55.

21. Gautron J, Hincke MT, Panheleux M, et al. Ovotransferrin is a matrix protein of the hen eggshell membranes and basal calcified layer. Conn Tissue Res. 2001;42:255-267.

22. Akagawa M, Wako Y, Suyama K. Lysyl oxidase coupled with catalase in egg shell membrane. Biochim Biophys Acta. 1999;1434(1): 151-160.

23. Hincke MT, Gautron J, Panheleux M, et al. Identification and localization of lysozyme as a component of eggshell membranes and eggshell matrix. Matrix Biol. 2000;19:443-453.

24. Ahlborn GJ, Clare DA, Sheldon BW, Kelly RW. Identification of eggshell membrane proteins and purification of ovotransferrin and $\beta$-NAGase from hen egg white. Protein J. 2006;25(1)71-81.

25. Systat Software, Inc., San Jose, CA. 2007. Accessed May 10, 2007. Available from: http://www.systat.com/.

Abstracts Service' (CAS), Scopus and the Elsevier Bibliographic databases. The manuscript management system is completely online and includes a very quick and fair peer-review system, which is all easy to use. Visit http://www.dovepress.com/testimonials.php to read real quotes from published authors. 